\title{
Modeling the Structural-Thermal-Electrical Coupling in an Electrostatically Actuated MEMS Switch and Its Impact on the Switch Stability
}

\author{
Hassen M. Ouakad ${ }^{1}$ and Mohammad I. Younis ${ }^{2}$ \\ ${ }^{1}$ Mechanical Engineering Department, King Fahd University of Petroleum and Minerals, Dhahran 31261, Saudi Arabia \\ ${ }^{2}$ Mechanical Engineering Department, State University of New York at Binghamton, Binghamton, NY 13902, USA
}

Correspondence should be addressed to Hassen M. Ouakad; houakad@kfupm.edu.sa

Received 22 February 2013; Revised 6 July 2013; Accepted 30 July 2013

Academic Editor: Eihab M. Abdel-Rahman

Copyright (C) 2013 H. M. Ouakad and M. I. Younis. This is an open access article distributed under the Creative Commons Attribution License, which permits unrestricted use, distribution, and reproduction in any medium, provided the original work is properly cited.

\begin{abstract}
Modeling and analysis for the static behavior and collapse instabilities of a MEMS cantilever switch subjected to both electrical and thermal loadings are presented. The thermal loading forces can be as a result of a huge amount of switching contact of the microswitch. The model considers the microbeam as a continuous medium and the electric force as a nonlinear function of displacement and accounts for its fringing-field effect. The electric force is assumed to be distributed over specific lengths underneath the microbeam. A boundary-value solver is used to study the collapse instability, which brings the microbeam from its unstuck configuration to touch the substrate and gets stuck in the so-called pinned configuration. We have found negligible influence of the temperature on the static stability of the switch. We then investigate the effect of the thermal heating due to the current flow on the cantilever switch while it is in the on position (adhered position). We also found slight effect on the static stability of the switch.
\end{abstract}

\section{Introduction and Background}

MEMS switches have attracted increasing interest in recent years because of their fast response, low power consumption, and low cost. Add to that, their simple fabrication process makes them easy to be fabricated at a low cost and hence commercialized easily. These switches have shown interest of wide spectrum of technologies such as mass/gas sensors, filters, switches, resonators, communication and radar systems, and recently cell phones. The basic structures that are used numerously to build MEMS and NEMS devices are principally made of cantilever or clamped-clamped beams.

Nevertheless, those structures can face several unwanted conditions while in use. They can stick to their lower electrodes or substrate due to several factors such as humidity due to large capillary forces [1-11], variation of its surrounding temperature, or some initial disturbances, such as mechanical shocks [12-15]. For example, humidity induces large capillary forces that a beam may undergo before it sticks to the substrate. Temperature variation creates instability in the microbeam that brings it to the state that its resorting force cannot resist this instability, and hence it collapses hitting the substrate. This is similar to the "pull-in" instability when the microbeam is actuated by electrostatic forces [14-18]. In fact, electrostatic actuation is one of the most common and preferred methods of actuation for microswitches. In this method, a microbeam forms one side of a capacitor. Underneath the microbeam is a stationary electrode forming the other side of the capacitor. By applying a DC load between the two electrodes, an attractive force is generated that deflects the microbeam toward the stationary electrode. If the electrostatic force is large enough, it will overcome the restoring force of the microbeam pulling it down toward the other electrode, which is called the pull-in phenomenon. Capacitive switches utilize pull-in to close or break an electric circuit in fast speed and short time [19].

When a cantilever microbeam collapses due to pull-in, its tip makes a contact with the substrate first where it gets 
stuck and becomes pinned to it. The rest of the microbeam remains separated from the substrate. This configuration is called the pinned or arc shape. If the DC voltage is too high, then the microbeam will undergo another collapse instability from the arc-shape to the so-called S-shape, where part of the microbeam will get adhered or stuck to the substrate. This configuration can cause troubles since the adhesion forces between the microbeam and the substrate can be very strong preventing the microbeam to come back to its original position when the DC load is set to zero. Also, it can cause undesirable contact with the gate circuit. Hence, it is very important to analyze the stability of the microbeam while it is on the arc configuration to make sure it will not get stuck to in the S-shape configuration or it will not go back to the unactuated (straight) shape. Some group [20-22] investigated experimentally and theoretically the transition of microbeams from the arc-shape pattern to the S-shape configuration when they are under the effect of capillary forces. For the case of electrically actuated structure, many groups revealed that the arc-shape and S-shape represent two stable configurations that may exist beyond the pull-in instability. However, and according to some investigations $[23,24]$, when a microswitch comes into contact with its actuating electrode, an electric current flows across the contact interface. The Joule heat generated when the current is passed through the microswitch causes its temperature to reach huge temperature making the contact region to wear at an increased rate and, at the extreme, to weld. However, the spring constant (mechanical stiffness) of the microswitch (cantilever) can prevent formation of the weld. The force with which the cantilever comes back to its "open" position has to be sufficient to break the weld. In some applications, the cantilever switch is desirable to remain in the "on" position in the arc configuration for a long time to pass current from a power source to an external electric circuit. In such cases, the beam will be heated up due to the passing of current through Joule heating. The generated heat affects the stiffness of the microbeam and may affect its stability; that is, the microbeam may pull up to the "off" position due to heat generation [25, 26]. Also, the resistance of the microbeam against dynamic disturbances, such as those due to shock and vibration, might be affected. Analyzing such a problem requires a multiphysics model that accounts for the coupling among the structural domain of the microbeam, the electrostatic domain due to DC actuation, the thermal domain due to heat generation, and the electrical domain due to the passing of current. The objective of this investigation is to present such a model and to study it thoroughly.

\section{Effect of Applied DC Voltage on the Static Stability of the Switch: The Floating Configuration (Switch OFF)}

In this section, we study the effect of applying a uniform temperature $T_{0}$ on the stability of the switch and how this can influence its collapse instability threshold (pull-in voltage). We consider first the floating configuration of the switch, Figure 1, in which the electrostatic force is distributed over the two considered gates.

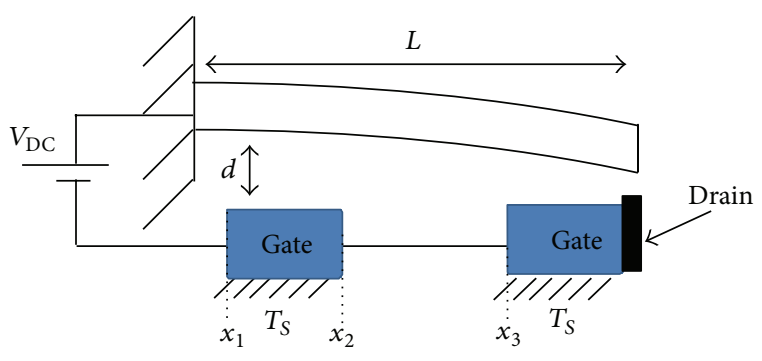

FIGURE 1: Schematic of the switch in the floating configuration (switch OFF).

TABLE 1: Geometrical and thermomechanical properties of the switch.

\begin{tabular}{lc}
\hline Young's modulus $E$ & $78 \mathrm{GPa}$ \\
Coefficient of thermal expansion $\alpha$ & $14 \times 10^{-6} \mathrm{~K}^{-1}$ \\
Thermal conductivity $k$ & $318 \mathrm{~W} / \mathrm{mK}$ \\
Length & $50 \mu \mathrm{m}$ \\
Thickness & $1.5 \mu \mathrm{m}$ \\
Gap & $1.52 \mu \mathrm{m}$ \\
Substrate temperature $T_{S}$ & $40^{\circ} \mathrm{C}$ \\
$x_{1}$ & $4 \mu \mathrm{m}$ \\
$x_{2}$ & $13 \mu \mathrm{m}$ \\
$x_{3}$ & $38 \mu \mathrm{m}$ \\
\hline
\end{tabular}

The static equation and boundary conditions of the switch due to the electrostatic forces and considering the fringing effect can be written as follows:

$$
\begin{gathered}
E I \frac{\partial^{4} w}{\partial x^{4}}=\frac{\varepsilon_{0} b V_{\mathrm{DC}}^{2}}{(d-w)^{2}}\left(1+0.65 \frac{(d-w)}{b}\right) F(x), \\
w(0)=0, \quad \frac{\partial w}{\partial x}(0)=0, \\
\frac{\partial^{2} w}{\partial x^{2}}(L)=0, \quad \frac{\partial^{3} w}{\partial x^{3}}(L)=0,
\end{gathered}
$$

where $F(x)=H\left(x-x_{1}\right)-H\left(x-x_{2}\right)+H\left(x-x_{3}\right)$ and $H$ is the unit step function (this term accounts for the discontinuity of the electrostatic field due to the two gates). For all the simulations in this paper, we considered the following cantilever microbeam parameters, Table 1.

As seen in (1), there is no explicit dependence on the static behavior on temperature due to the fact that the microbeam can expand freely when heated. Hence, there is no induced stress due to thermal heating. Instead, there is induced strain and change in the length of the beam.

We first simulate the response of the microbeam to electrostatic load alone (without any temperature effect) up to the pull-in instability. We used the beam model, (1) and (2), which have been solved numerically using a boundary value problem (BVP) solver in MATLAB. We also build and use a coupled multiphysics finite-element model in ANSYS to validate our analytical predictions. Figure 2 shows the static deflection of the switch using the analytical model (solid line) and using the finite element model (circles). 


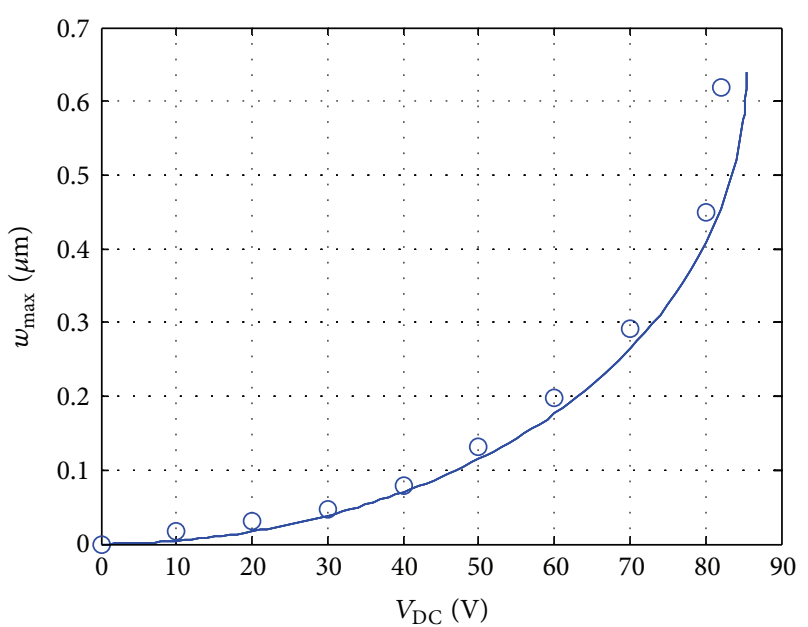

(a)

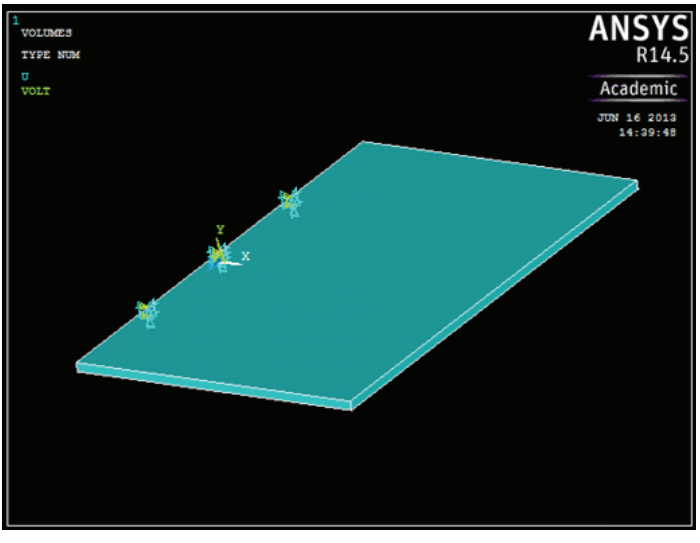

(b)

Figure 2: (a) The switch static deflection versus the applied DC voltage using the analytical model (solid blue line) and the finite element model (circles). (b) The FE model in ANSYS.

The figure shows full agreement among the results of both approaches. We obtained a pull-in voltage approximately equal to 85.5 Volt using the analytical model and 82 Volt using the ANSYS FE model. Note that in the FE model, we use the SOLID95 structural brick element to model the microbeam and a coupled field element to model the electrical-structural coupling.

\section{The Effect of Induced Temperature due to Current Flow on the Static Stability of the Switch: The Pinned Configuration (Switch ON)}

In this section, we investigate the effect of thermal heating due to the current flow in the actuated cantilever microbeam on its structural stability. An analytical model is used for this investigation.

In the below, $T(x)$ refers to the temperature difference between the microbeam and the substrate, which is assumed fixed at $40^{\circ} \mathrm{C}$. The static equations of the coupled structuralthermal problem when the switch is in the pinned configuration, Figure 3, can be written as follows [27, 28]:

$$
\begin{aligned}
E I \frac{d^{4} w}{d x^{4}}= & \frac{\alpha E b h^{2}}{4} \frac{d^{2} T}{d x^{2}}+\frac{\varepsilon_{0} b V_{\mathrm{DC}}^{2}}{(d-w)^{2}} F(x) \\
& +\left[\frac{E A}{2 L} \int_{0}^{L}\left(\frac{d w}{d x}\right)^{2} d x-\alpha E A T\right] \frac{d^{2} w}{d x^{2}}
\end{aligned}
$$

(beam equation)

$$
\frac{d}{d x}\left(k(T) \frac{d T}{d x}\right)+\frac{\rho(T) I^{2}}{A^{2}}=\frac{S k_{a}}{h d} T,
$$

(heat conduction equation).

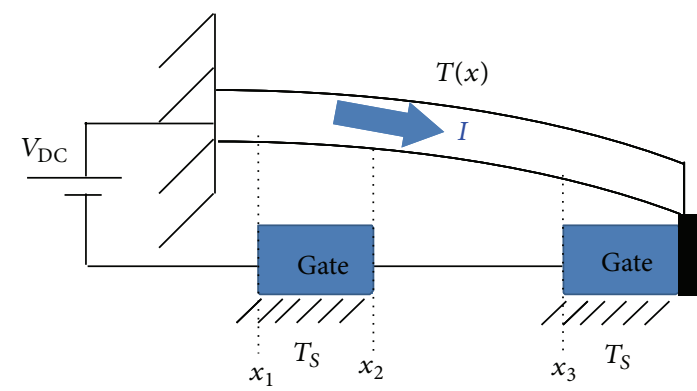

FIGURE 3: Schematic of the switch in the pinned configuration (switch ON).

In (3b), $S$ is a shape factor that accounts for the conduction from the vertical faces of the microbeam to the substrate, $k_{a}$ is the air thermal conductivity, $I$ stands for the current passing through the microbeam, $k(T)$ and $\rho(T)$ are the thermal conductivity and the resistivity of the microbeam, respectively, which depend on the temperature as follows [29]:

$$
\begin{gathered}
k(T)=\frac{1}{\left(-10^{-5} T(x)+0.014\right)}, \\
\rho(T)=2.44 \times 10^{-8}\left(1+3.7 \times 10^{-3} T(x)\right) .
\end{gathered}
$$

Equation (3a) represents a nonlinear static equation for the microbeam accounting for its geometric nonlinearity due to mid-plane stretching effect (the integral term in the equation), the electrostatic force, and the induced thermal stress.

From (3b), we solve analytically for the expression of the temperature variation along the length of the microbeam. Then we feed this expression into the beam equation, (3a), and solve for the static profile $w(x)$ of the switch using the boundary-value solver in MATLAB. 


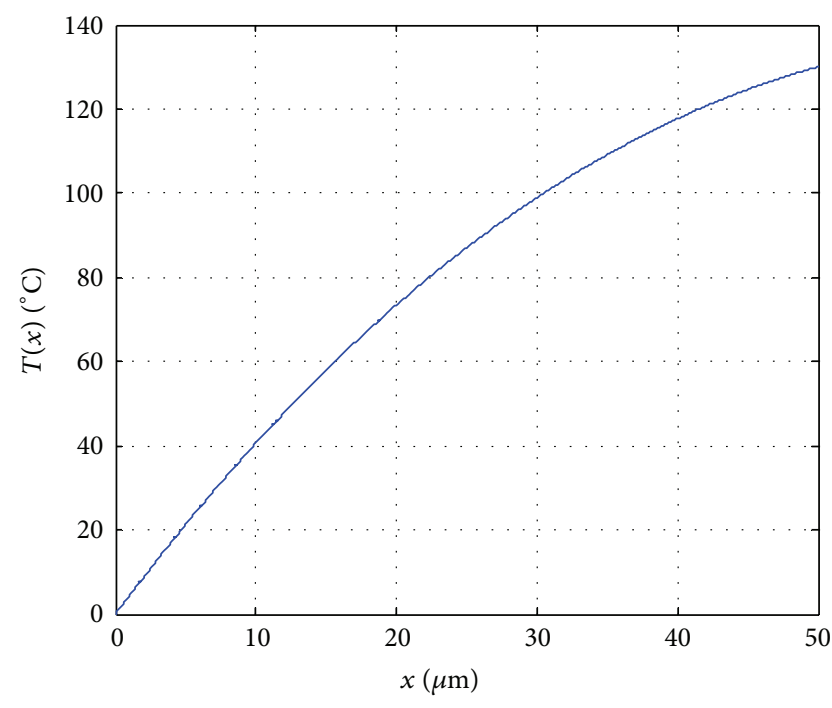

FIgURE 4: The temperature profile of the switch for $I=4$ A.

The switch deflection $w$ must satisfy the following boundary conditions:

$$
\begin{array}{ll}
w(0)=0, & \frac{\partial w}{\partial x}(0)=0, \\
w(L)=d, & \frac{\partial^{2} w}{\partial x^{2}}(L)=0 .
\end{array}
$$

And the temperature distribution must satisfy the following boundary conditions:

$$
T(0)=0, \quad T(L)=T(I) .
$$

Here $T(I)$ is the temperature boundary which depends on the current passing through the microbeam. This value can be obtained from a thermal finite-element simulation model. In this investigation, we used the temperature readings provided from a thermal finite-element simulation model [30]. For the simulated case, we considered the case of $I=4 \mathrm{~A}$ in which case the end temperature is taken to be $170^{\circ} \mathrm{C}$ so that we took $T(L)=130^{\circ} \mathrm{C}$. Figure 4 shows the temperature profile of the switch considering a $4 \mathrm{~A}$ current passing through the crosssectional area of the switch.

We use this temperature profile in the beam equation and then solved the resulting equation for the static deflection of the microbeam. Figure 5 shows the static deflection profile of the microbeam considering the effect of the temperature and compares it to the case of neglecting the thermal effect. As seen in the figure, the effect of temperature seems to have effect on the static profile of the microbeam and, hence, on the stability of the switch.

Figure 6 displays the static deflection profile of the switch considering the effect of the temperature and varying the temperature condition and current at its tip. As seen in the figure, the effect of increasing the temperature, that is, the current, seems to have also an effect on the static profile of the microbeam and, hence, the possibility for the switch to pull up.

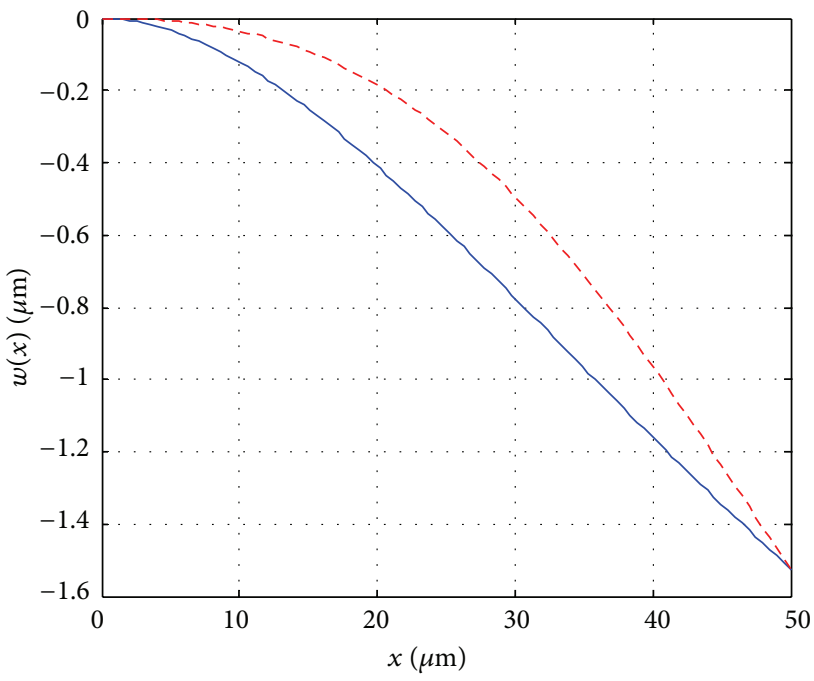

Without the effect of temperature

- - With the effect of temperature

FIgURE 5: The static profile of the switch for $\mathbf{V}_{\mathrm{DC}}=90 \mathrm{Volt}, I=$ $4 \mathrm{~A}$ with and without considering the effect of temperature, that is, $(T(x) \neq 0$, and $T(x)=0$, resp.).

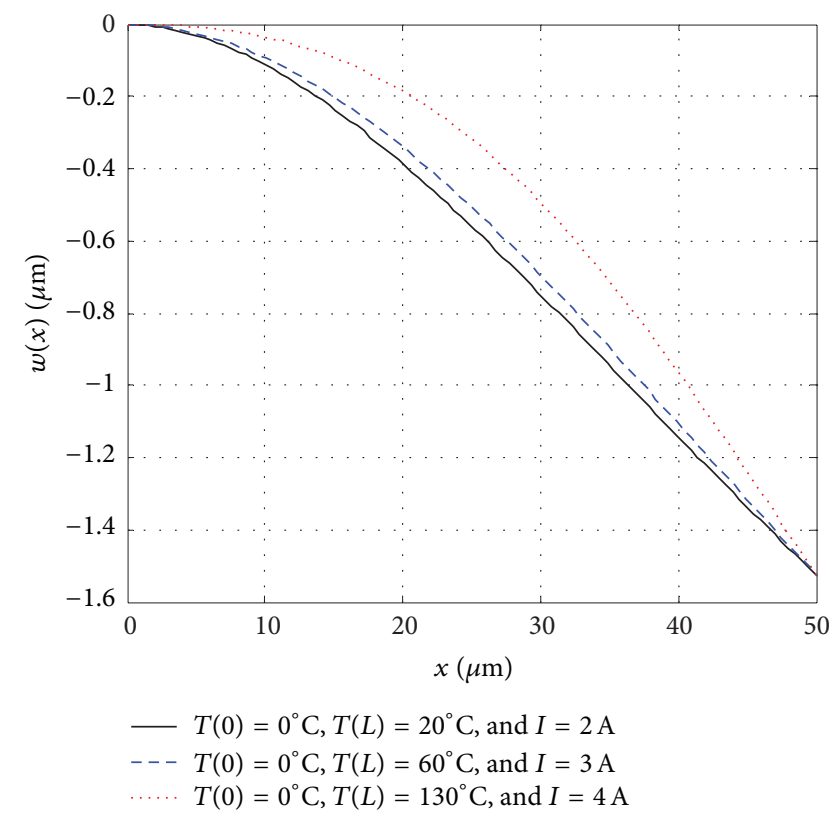

FIgURE 6: The static profile of the switch for $\mathbf{V}_{\mathrm{DC}}=90$ Volt, with considering the effect of temperature and for various temperature conditions at the switch tip.

\section{The Effect of Applying a Constant Temperature along the Beam on Its Static Stability in the Pinned Configuration (Switch ON)}

To gain more insight into the influence of the temperature variation on the static behavior of the switch when it is in the 


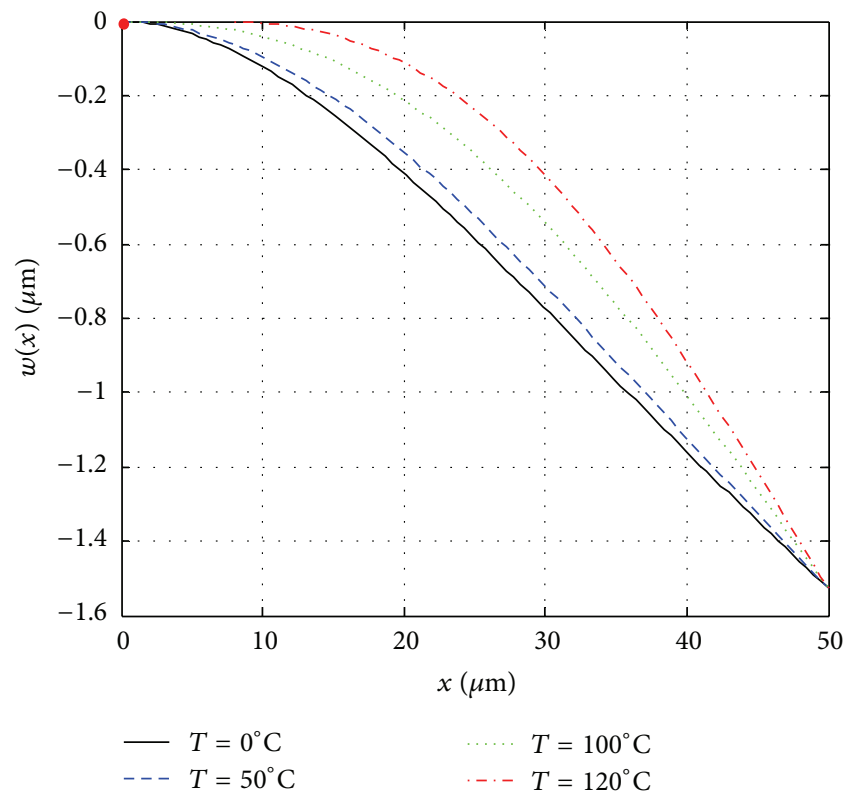

(a)

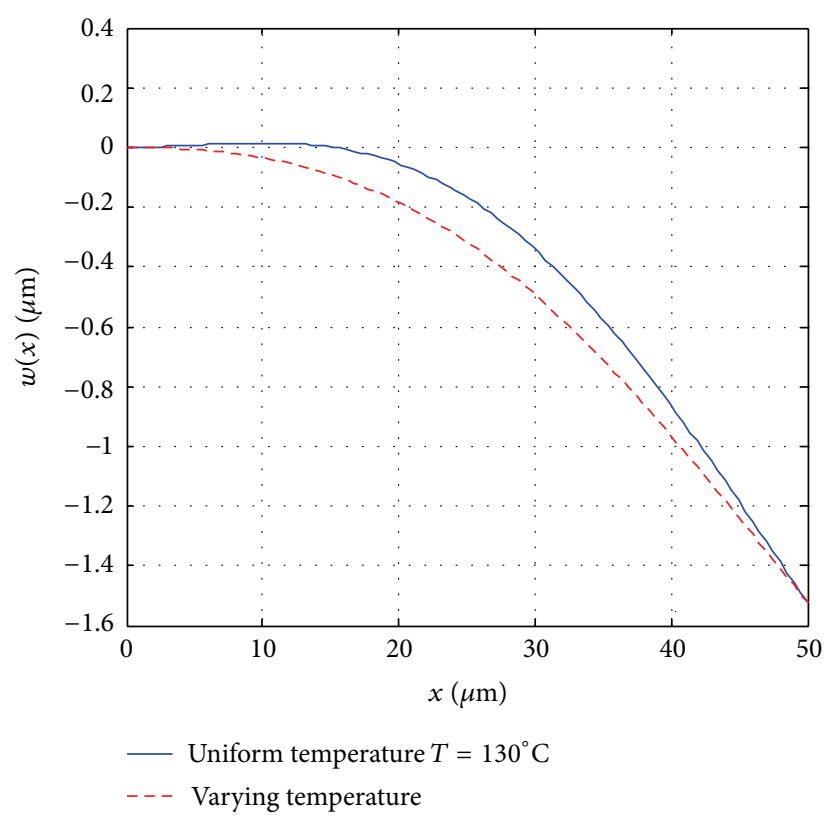

(b)

Figure 7: (a) The switch static profile for different uniform temperature variations, and (b) comparison between the static profile of the switch considering a $130^{\circ} \mathrm{C}$ uniform variation temperature (solid line) and considering the induced temperature variation with $T(L)=130^{\circ} \mathrm{C}$ (dashed line).

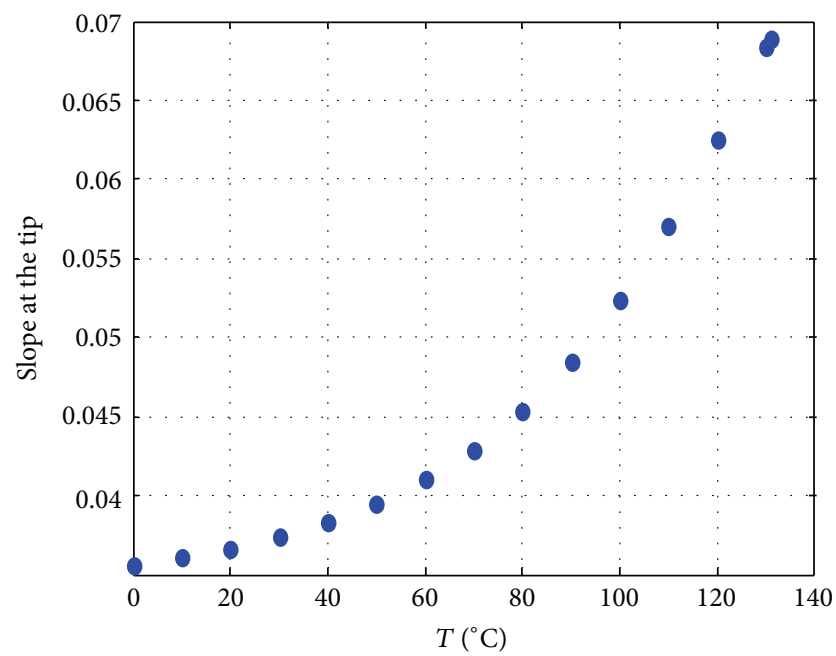

FIGURE 8: Variation of the slope at the switch tip versus the uniform temperature variation.

ON position, we assume a constant applied temperature along the beam length $(T(x)=$ constant $)$ and examine the influence of increasing and decreasing it on the switch.

Figure 7(a) shows the influence of varying the temperature distribution on the switch profile. As noted, the beam is deformed away from the substrate with the increase in temperature. Figure 7(b) compares the profile in this case with the case of allowing variation in the temperature along the beam length due to the passing of current, as in Section 3 .

Figure 8 shows the variation of the switch tip slope versus the temperature variation. The slope at the switch tip increases with the increase of the temperature variation. We notice that at the value $T \approx 132^{\circ} \mathrm{C}$, the slope goes to infinity indicating the possibility that the switch undergoes buckling instability, that is; it pulls up.

To ascertain the instability, we solve the linear eigen value problem of the microbeam in the pinned configuration. For this, we derive first the linear eigen value problem (freevibration) of the considered switch. We first split the response into a static component due to the DC voltage, denoted by $w_{s}(x)$, and a dynamic component denoted by $w_{d}(x, t)$; that is,

$$
w(x, t)=w_{s}(x)+w_{d}(x, t)
$$

Substituting (7) into (1) and (5), adding the inertia term, and retaining only the linear terms in $w_{d}$ yield the following equations describing the small free vibration of the switch around its static equilibrium position:

$$
\begin{aligned}
& E I \frac{\partial^{4} w_{d}}{\partial x^{4}}+\rho A \frac{\partial^{2} w_{d}}{\partial t^{2}} \\
& \quad=\varepsilon_{0} b V_{\mathrm{DC}}^{2}\left(\frac{2+(0.65 / b)\left(d-w_{s}\right)}{\left(d-w_{s}\right)^{2}} F(x)\right) w_{d}, \\
& w_{d}(0, t)=0, \quad \frac{\partial w_{d}}{\partial x}(0, t)=0, \\
& \frac{\partial^{2} w_{d}}{\partial x^{2}}(L, t)=0, \quad \frac{\partial^{3} w_{d}}{\partial x^{3}}(L, t)=0 .
\end{aligned}
$$




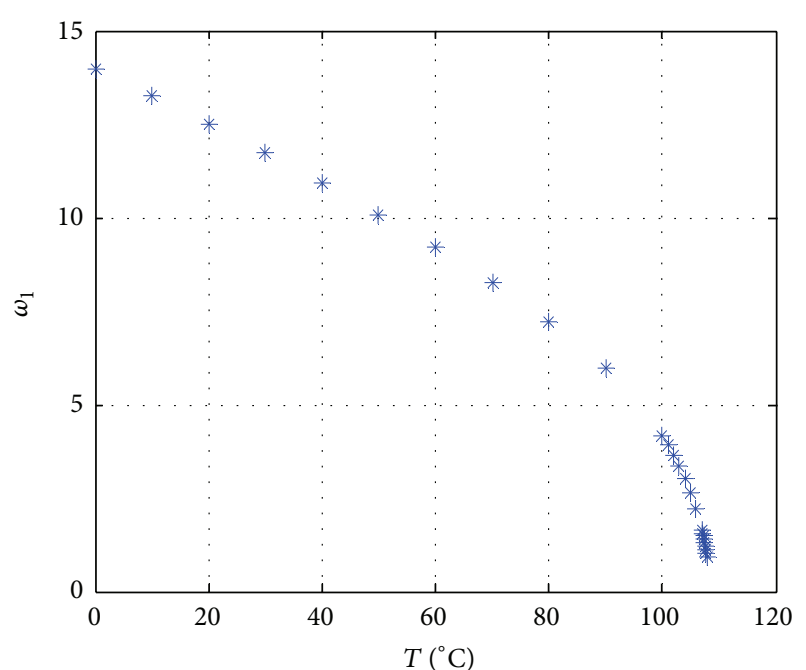

FIGURE 9: Variation of the normalized fundamental natural frequency $\omega_{1}$ with the uniform temperature variation without the stretching effect.

To derive the eigenvalue problem, and hence, obtain the corresponding natural frequencies of the switch for a given DC voltage or constant temperature, we let

$$
w_{d}(x, t)=\phi(x) e^{i \omega t},
$$

where $\phi(x)$ is the mode shape and $\omega$ is its corresponding nondimensional natural frequency. Substituting (9) into (8) yields the following eigenvalue problems for the switch:

$$
\begin{gathered}
E I \phi^{i v}(x)-\rho A \omega^{2} \phi(x) \\
=\varepsilon_{0} b V_{\mathrm{DC}}^{2}\left(\frac{2+(0.65 / b)\left(d-w_{s}\right)}{\left(d-w_{s}\right)^{2}} F(x)\right) \phi(x), \\
\phi(0)=\phi^{\prime}(0)=0, \quad \phi^{\prime \prime}(L)=\phi^{\prime \prime \prime}(L)=0,
\end{gathered}
$$

where the prime above refers to a derivative with respect to $x$. To obtain the linear natural frequency $\omega$ of the considered carbon nanotube, we first solve for the static deflection $w_{s}$ as was done in Section 2. Then, we solve (10) for the linear natural frequency $\omega$ using a shooting method combined with an iterative scheme until convergence [14]. Note that at any structural instability of the switch, its linear natural frequency (eigenvalue) drops to zero [31].

Figure 9 shows the normalized natural frequency with the applied uniform temperature. We notice in the figure a continuous decrease in the natural frequency until it reaches zero at the instability.

\section{Effect of Uniform Temperature on the Static Stability of the Switch Considering a Clamped Configuration at the Tip (Switch ON)}

In this section, we consider also the influence of a uniform temperature variation on the static behavior of the switch

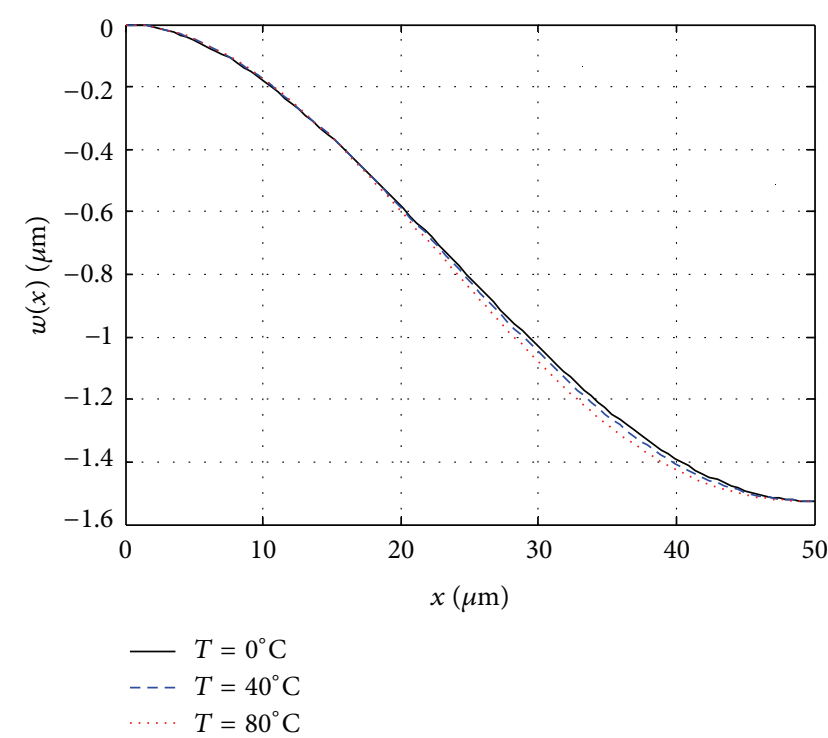

FIGURE 10: The switch deflection versus the normalized beam length for different uniform temperature variations.

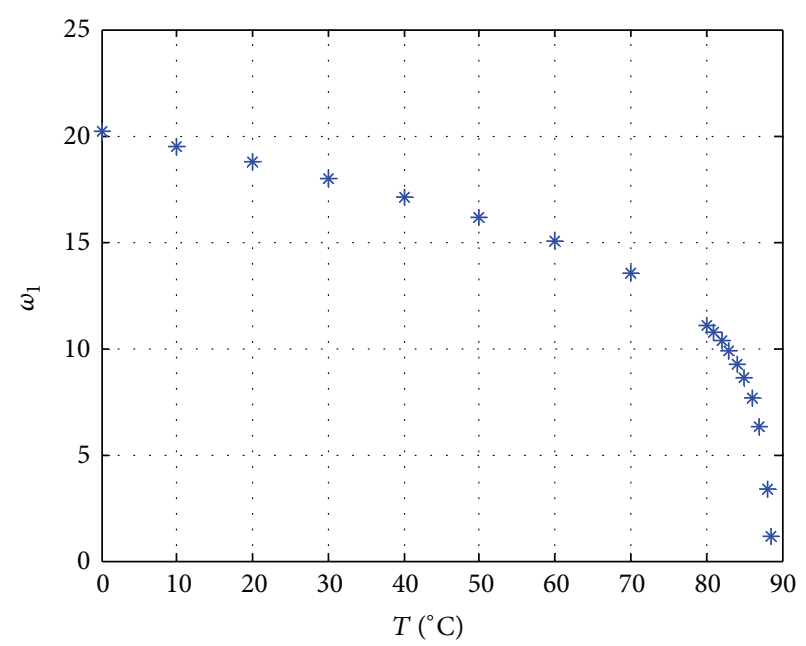

FIGURE 11: Variation of the normalized fundamental natural frequency $\omega_{1}$ with the uniform temperature variation without the stretching effect.

when considering a clamped condition at the tip rather than the pinned one adopted in Sections 2 and 3. The influence of varying the temperature distribution on the switch profile is illustrated in Figure 10. Notice that the switch here pulls down when we increase the temperature value until reaching instability; that is, reaching the adhered configuration, at a value of temperature difference $T$ equal to $97^{\circ} \mathrm{C}$. In this configuration, a portion of the beam toward the tip will get stuck to the substrate or the gate.

The same as we did for the pinned configuration, we solve here also the linear eigen value problem of the microbeam while considering the clamped configuration. We notice from Figure 11 also a continuous decrease in the natural frequency until it reaches zero at the instability. 


\section{Conclusions}

In this paper, the stability of a cantilever switch under electric and thermal loads was investigated. The temperature effect on the cantilever microbeam while it is in the off position (unactuated) and its influence on its instability threshold (pull-in voltage/gate actuation threshold) were described and quantified. We have found negligible influence of the temperature on the static stability of the cantilever microbeam. We then investigate the effect of the thermal heating due to the current flow on the cantilever switch while it is in the on position. We also found slight effect on the static stability of the switch.

At this stage of research, an experimental investigation can be very useful to assess the validity and accuracy of our models.

\section{References}

[1] T. Yu, R. Ranganathan, N. Johnson, N. Yadav, R. Gale, and T. Dallas, "In situ characterization of induced stiction in a MEMS," Journal of Microelectromechanical Systems, vol. 16, no. 2, pp. 355-364, 2007.

[2] A. A. Savkar, K. D. Murphy, Z. C. Leseman, T. J. Mackin, and M. R. Begley, "On the use of structural vibrations to release stiction failed MEMS," Journal of Microelectromechanical Systems, vol. 16, no. 1, pp. 163-173, 2007.

[3] Z. C. Leseman, S. P. Carlson, and T. J. Mackin, "Experimental measurements of the strain energy release rate for stictionfailed microcantilevers using a single-cantilever beam peel test," Journal of Microelectromechanical Systems, vol. 16, no. 1, pp. 3843, 2007.

[4] T. Lam and R. B. Darling, "Physical modeling of MEMS cantilever beams and the measurement of stiction force," Journal of Modelling and Simulation of Microsystems, vol. 4, pp. 418-421, 2001.

[5] L. L. Mercado, S. Kuo, T. T. Lee, and L. Liu, "Mechanicsbased solutions to RF MEMS switch stiction problem," IEEE Transactions on Components and Packaging Technologies, vol. 27, no. 3, pp. 560-567, 2004.

[6] W. M. van Spengen, R. Puers, and I. de Wolf, "The prediction of suction failures in MEMS," IEEE Transactions on Device and Materials Reliability, vol. 3, no. 4, pp. 167-172, 2003.

[7] O. Raccurt, F. Tardif, F. A. D’Avitaya, and T. Vareine, "Influence of liquid surface tension on stiction of SOI MEMS," Journal of Micromechanics and Microengineering, vol. 14, no. 7, pp. 10831090, 2004.

[8] C. H. Mastrangelo, Suppression of Stiction in MEMS, Center for Integrated Microsystems, Department of Electrical Engineering and Computer Science, University of Michigan, Ann Arbor, Mich, USA, 2000.

[9] M. P. de Boer and T. A. Michalske, "Accurate method for determining adhesion of cantilever beams," Journal of Applied Physics, vol. 86, no. 2, pp. 817-827, 1999.

[10] M. P. de Boer, P. J. Clews, B. K. Smith, and T. A. Michalske, "Adhesion of polysilicon microbeams in controlled humidity ambients," Materials Research Society Symposium Proceedings, vol. 518, pp. 131-136, 1997.

[11] M. Sheehy, J. Punch, S. Goyal, M. Reid, M. Lishchynska, and G. Kelly, "The failure mechanisms of micro-scale cantilevers under shock and vibration stimuli," Strain, vol. 45, no. 3, pp. 283-294, 2009.

[12] F. M. Alsaleem, M. I. Younis, and M. I. Ibrahim, "A study for the effect of the PCB motion on the dynamics of MEMS devices under mechanical shock," Journal of Microelectromechanical Systems, vol. 18, no. 3, pp. 597-609, 2009.

[13] H. Ouakad, M. Younis, and F. AlSaleem, "Dynamic response of an electrostatically actuated microbeam to drop-table test," Journal of Micromechanics and Microengineering, vol. 22, no. 9, Article ID 095003, 7 pages, 2012.

[14] E. M. Abdel-Rahman, M. I. Younis, and A. H. Nayfeh, "Characterization of the mechanical behavior of an electrically actuated microbeam," Journal of Micromechanics and Microengineering, vol. 12, no. 6, pp. 759-766, 2002.

[15] S. Gorthi, A. Mohanty, and A. Chatterjee, "Cantilever beam electrostatic MEMS actuators beyond pull-in," Journal of Micromechanics and Microengineering, vol. 16, no. 9, pp. 18001810, 2006.

[16] J. A. Knapp and M. P. de Boer, "Mechanics of microcantilever beams subject to combined electrostatic and adhesive forces," Journal of Microelectromechanical Systems, vol. 11, no. 6, pp. 754764, 2002.

[17] H. A. C. Tilmans and R. Legtenberg, "Electrostatically driven vacuum-encapsulated polysilicon resonators-part II: theory and performance," Sensors and Actuators A, vol. 45, no. 1, pp. 67-84, 1994.

[18] Y. Zhang and Y. Zhao, "Static study of cantilever beam stiction under electrostatic force influence," Acta Mechanica Solida Sinica, vol. 17, no. 2, pp. 104-112, 2004.

[19] M. I. Younis, E. M. Abdel-Rahman, and A. Nayfeh, "A reducedorder model for electrically actuated microbeam-based MEMS," Journal of Microelectromechanical Systems, vol. 12, no. 5, pp. 672-680, 2003.

[20] C. H. Mastrangelo and C. H. Hsu, "Simple experimental technique for the measurement of the work of adhesion of microstructures," in Proceedings of the IEEE Solid-State Sensor and Actuator Workshop, pp. 208-212, June 1992.

[21] M. P. de Boer, P. J. Clews, B. K. Smith, and T. A. Michalske, "Adhesion of polysilicon microbeams in controlled humidity ambients," in Proceedings of the Materials Research Society Symposium, vol. 518, pp. 131-136, San Francisco, Calif, USA, April 1998.

[22] H. M. Ouakad and M. I. Younis, "Modeling and simulations of collapse instabilities of microbeams due to capillary forces," Mathematical Problems in Engineering, vol. 2009, Article ID 871902, 16 pages, 2009.

[23] Tyco, Relay Contact Life, Application Note 13C3236, Tyco Electronics, Corporation-P\&B, Winston-Salem, NC, USA, 2000.

[24] Tyco, Contact Arc Phenomenon, Application Note 13C3203, Tyco Electronics, Corporation-P\&B, Winston-Salem, NC, USA, 2000.

[25] R. P. Hennessy, A. Basu, G. G. Adams, and N. E. McGruer, "Hotswitched lifetime and damage characteristics of MEMS switch contacts," vol. 23, no. 5, Article ID 055003, 11 pages, 2013.

[26] M. S. Machate, Joule heat effects on reliability of RF MEMS switches [M.S. thesis], Worcester Polytechnic Institute, Mechanical Engineering, 2003.

[27] L. C. Wu and K. M. Chang, "Thermoelastic coupled modeling for a thermal bimorph actuator," Mechanics Research Communications, vol. 34, no. 7-8, pp. 553-560, 2007. 
[28] B. A. Boley and J. H. Weiner, Theory of Thermal Stresses, John Wiley \& Sons, New York, NY, USA, 1960.

[29] http://www.matweb.com.

[30] A. S. Daley, E. Ahmed, W. J. Isaac, S. Kanakasabapathi, K. C. Fred, and G. A. Virupaksha, "Power overlay structure of an mems device and method for manufacturing the same by employing," Korean Patent\# 1020080012608, 2008.

[31] M. I. Younis, MEMS Linear and Nonlinear Statics and Dynamics, Springer, New York, NY, USA, 2011. 


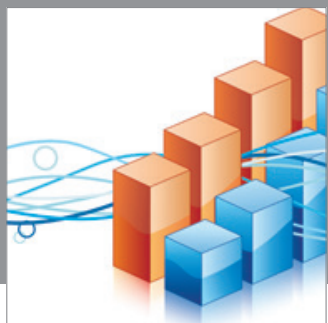

Advances in

Operations Research

mansans

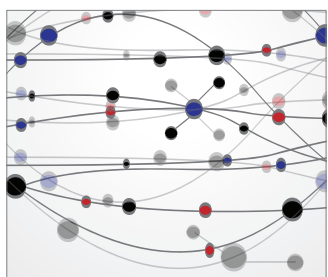

The Scientific World Journal
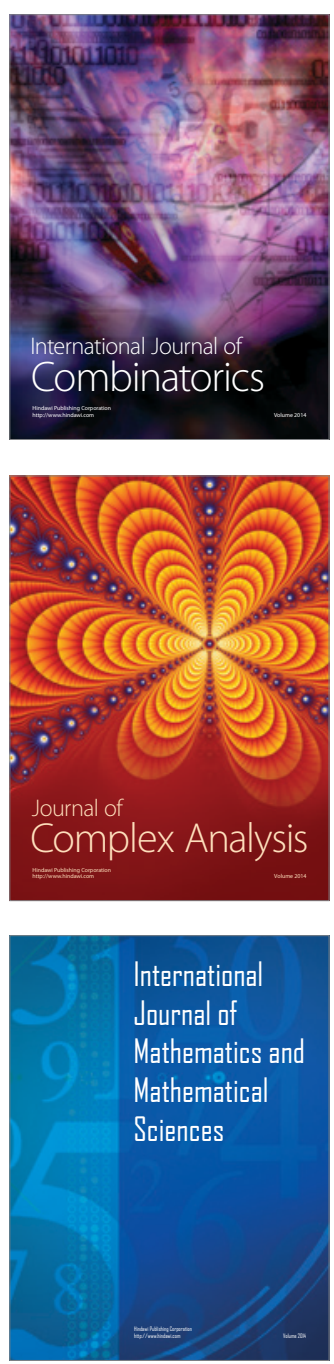
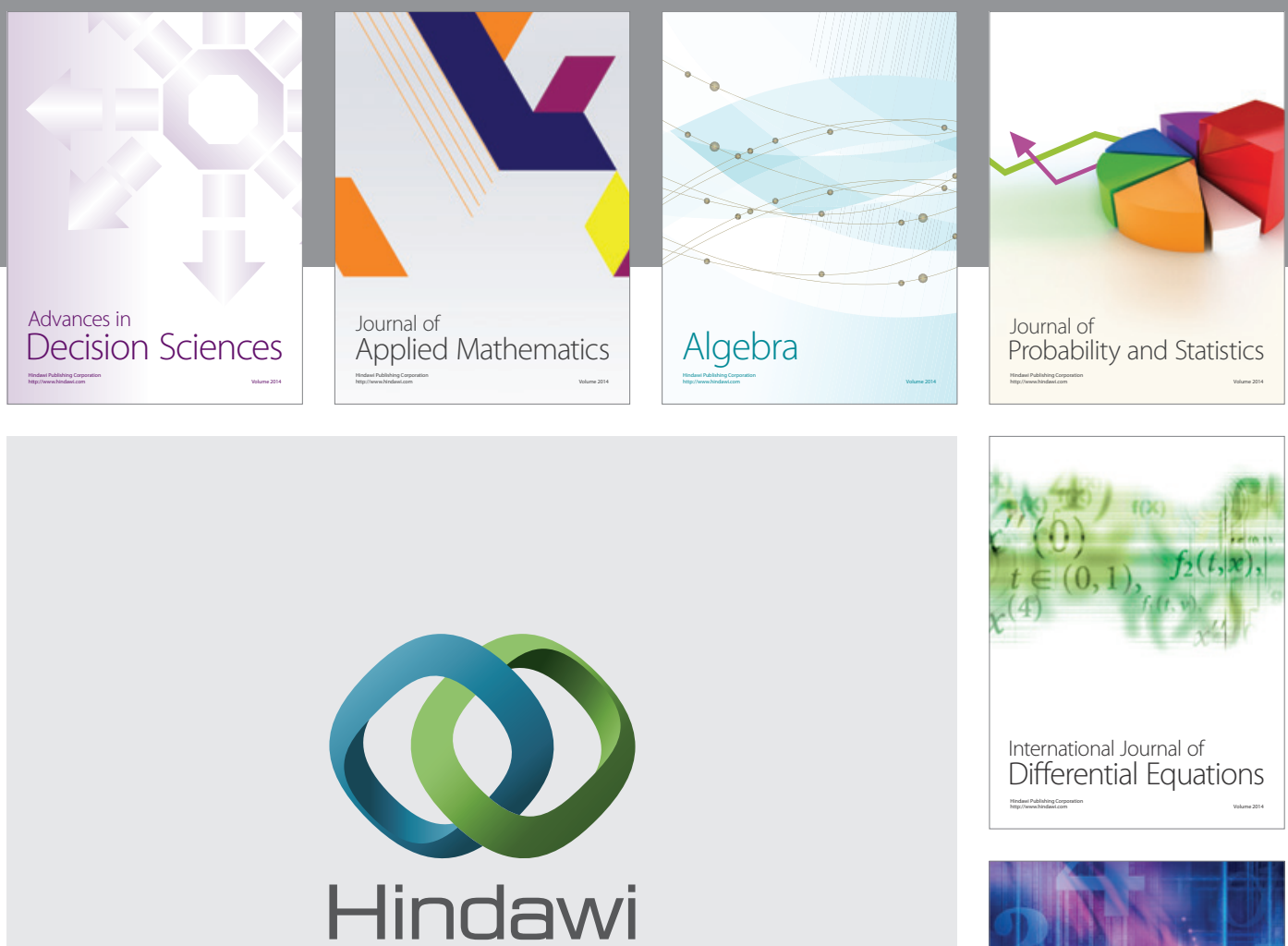

Submit your manuscripts at http://www.hindawi.com
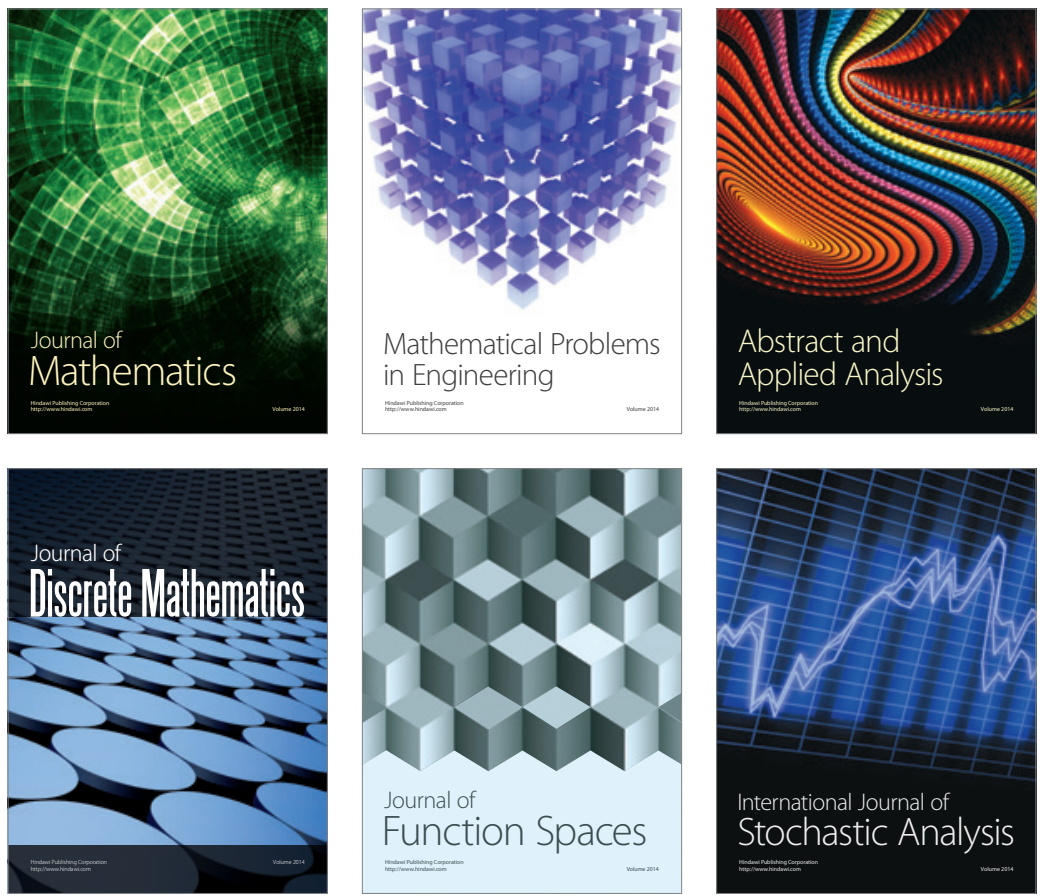

Journal of

Function Spaces

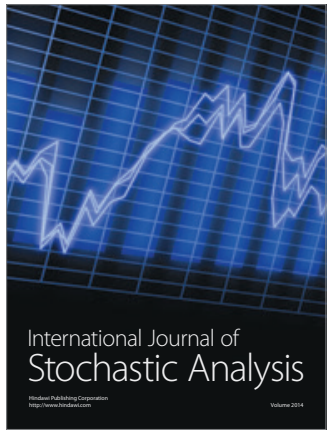

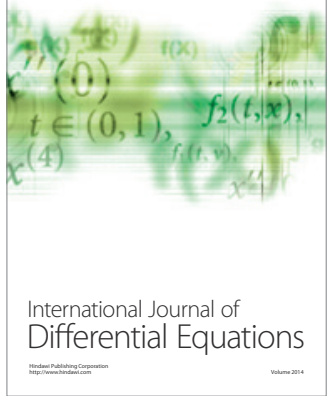
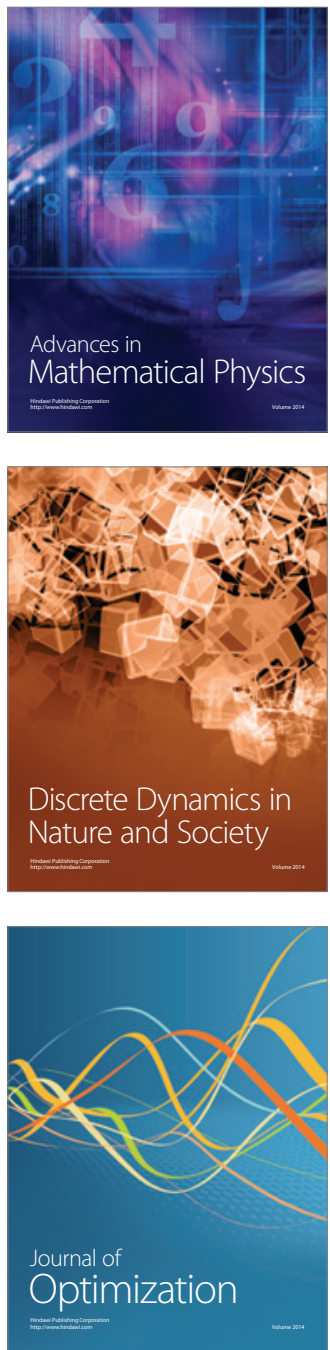